\title{
Plasma Levels of Interleukin 12 Family Cytokines and Their Relevant Cytokines in Adult Patients with Chronic Immune Thrombocytopenia before and after High-Dose Dexamethasome Treatment
}

\author{
Qingsheng $\mathrm{Li}^{\mathrm{a}}$ Linjie Zhang ${ }^{\mathrm{b}}$ Ruixiang $\mathrm{Xia}^{\mathrm{a}}$ Qingshu Zeng ${ }^{\mathrm{a}}$ \\ Yongqing Wang ${ }^{\mathrm{a}}{\text { Liang } \mathrm{Xia}^{\mathrm{a}} \text { Leiming } \mathrm{Xia}^{\mathrm{a}} \text { Mingzhen Yang }}^{\mathrm{a}}$ \\ ${ }^{a}$ Department of Hematology, The First Affiliated Hospital, and ${ }^{\mathrm{b}}$ Department of Immunology, Anhui Medical \\ University, Hefei, PR China
}

\section{Key Words}

Immune thrombocytopenia - Interleukin $12 \cdot$ Cytokines .

Dexamethasone

\begin{abstract}
Objective: We aimed to investigate the expression of interleukin 12 (IL-12) family cytokines (IL-12, IL-23, IL-27 and IL35 ) and their relevant cytokines (IFN- $\gamma$, IL-4, IL-17A and IL-10) in patients with chronic immune thrombocytopenia (cITP) as well as the effect of high-dose dexamethasone (HD-DXM) treatment on this expression. Materials and Methods: DXM was administered orally at a dose of $40 \mathrm{mg}$ per day for 4 consecutive days to 38 patients with CITP. We measured the plasma levels of IL-12p70, IL-23, IL-27, IFN- $\gamma$, IL-4 and IL-17A before and after treatment and also in 36 matched healthy controls, by means of FlowCytomix ${ }^{\mathrm{TM}}$ technology. The plasma levels of IL-10 and IL-35 were measured by enzymelinked immunosorbent assay. Results: Significantly higher plasma levels of IL-12p70, IL-23, IL-27, IFN- $\gamma$ and IL-17A were observed in cITP patients than in controls $(p<0.01)$, and after HD-DXM treatment, these levels decreased significantly $(p<$ 0.01 ). However, significantly lower plasma levels of IL-4, IL-10 and IL-35 were observed in CITP patients than in controls
\end{abstract}

$(p<0.01)$; after the HD-DXM treatment, these levels had increased significantly in the CITP patients $(p<0.01)$. Moreover, the cytokine levels of patients who attained a complete response returned to the levels of normal controls $(p>0.05)$ but were not corrected in the patients who had no response $(p<0.01)$. Conclusions: The patients with cITP had abnormal expression of the IL-12 family cytokines and their relevant cytokines levels, and HD-DXM treatment corrected the derangement of plasma cytokines. Measuring cytokine levels may help in the clinical assessment of cITP.

๑) 2015 S. Karger AG, Basel

\section{Introduction}

Chronic immune thrombocytopenia (cITP) is an autoimmune disease which prematurely destroys platelets, mainly in the spleen, liver and bone marrow [1]. The pathophysiology of cITP is heterogeneous and complex. Besides the well-known autoantibody-mediated destruction of platelets in the reticuloendothelial system [1], several T-cell abnormalities have been identified in cITP, including a polarization toward Thelper 1 (Th1) [2], changes in the number of regulatory $\mathrm{T}$ (Treg) cells and Th17 
cells $[3,4]$ and the destruction of platelets by cytotoxic T lymphocytes [5]. However, the mechanism of cellular immune abnormalities in patients with cITP remains unclear.

The IL- 12 cytokine family consists of IL-12, IL-23, IL27 and IL-35, which have diverse functions and play a role in both pro- and anti-inflammatory responses [6]. The IL-12 cytokine is composed of two protein subunits called p35 and p40, and is the first cytokine shown to be able to drive the differentiation of naïve $\mathrm{T}$ cells into Th1 cells, with high IFN- $\gamma$ production [7]. IL-23 is composed of a p40 subunit shared by the IL- 12 and p19 subunits. It not only acts on Th1 cells like IL-12, but has been associated with the generation of the Th17 response and the production of IL-17 [8]. IL-27 is composed of Epstein-Barr virusinduced gene 3, i.e. EBI-3, and a p28 subunit, which can synergize with IL-12 to promote IFN- $\gamma$ production early in the Th1 response [9]. IL-27 also acts in an anti-inflammatory manner, promoting induction of Foxp3-IL10-producing $\operatorname{Tr} 1$ cells [10] and inhibiting the differentiation of Th2 [11] and Th17 cells [12]. IL-35, the most recently identified member of this family, is composed of EBI-3 and p35 [13], and it has been considered to be a characteristic factor of Treg cells, like TGF- $\beta$ and IL-10 [14]. Additional evidence demonstrates that IL-35 is an important anti-inflammatory cytokine and can efficiently suppress the activity of CD4+ effector T cells (including Th1, Th2 and Th17) and reduce the progression of inflammatory and autoimmune diseases $[15,16]$.

Some reports have shown that high-dose dexamethasone (HD-DXM) might be a promising alternative to prednisone as a first- or second-line treatment for ITP patients $[17,18]$. HD-DXM could correct Th1 polarization by altering the Th1/Th2 cytokine profile [19]. IL-12 family cytokines appear to play an important role in determining the types of $\mathrm{T}$-cell response generated. However, the exact expression of IL-12 family cytokines and their relevant cytokines (IFN- $\gamma$, IL-4, IL-17A and IL-10) in cITP patients is not known. In this study, we investigated the expression of these cytokines in cITP and the possible effects of HD-DXM on these cytokines in cITP patients.

\section{Materials and Methods}

\section{Subjects}

From January 2010 to October 2013, 38 patients (25 females and 13 males, with an age range of 17-72 years) with cITP were enrolled in this study at the Department of Hematology, The First Affiliated Hospital, Anhui Medical University in Hefei, China. The platelet counts of the patients ranged from 2 to $47 \times 10^{9} / 1$ with a median of $11 \times 10^{9} / 1$. For at least 1 month beforehand, no patients had received any glucocorticoids (GCs) or other immunosuppressive treatments. Patients were excluded if they were pregnant or suffering from diabetes, hypertension, cardiovascular diseases, active or chronic infections or connective tissue diseases such as systemic lupus erythematosus. All the patients involved met the diagnostic criteria of cITP described previously [20]. The main features of the enrolled patients are presented in table 1 . The control group consisted of 36 sex- and age-matched healthy volunteers ( $25 \mathrm{fe}$ males and 11 males, with an age range of $21-59$ years). Their platelet counts ranged from 103 to $280 \times 10^{9} / 1$, with a median of $189 \times$ $10^{9} / 1$. The study was approved by the Ethics Committee of Anhui Medical University. Informed written consent was obtained from all the patients and healthy controls in accordance with the Declaration of Helsinki.

\section{Treatment Regimen}

All the patients received $40 \mathrm{mg}$ of oral DXM daily for 4 consecutive days. No maintenance or other treatment modality was used. An evaluation of the initial response was made at the end of the second week after treatment initiation. The response was evaluated according to the following criteria. A complete response was defined as a platelet count $\geq 100 \times 10^{9} / 1$ and the absence of bleeding; a response was defined as a platelet count between 30 and 100 $\times 10^{9} / 1$, at least a doubling of the baseline counts and the absence of bleeding; no response was defined as any platelet count $<30 \times$ $10^{9} / 1$ and less than a doubling of the baseline counts or bleeding.

\section{Immunoassays to Quantify Cytokines with FlowCytomix Kits}

Peripheral blood was collected into heparin-anticoagulant vacutainer tubes before and 2 weeks after the treatment with HDDXM [21]. Plasma was obtained from all subjects by centrifugation and stored at $-80^{\circ} \mathrm{C}$ to determine the cytokines. Eight different cytokines were examined in the cITP patients and normal controls. The concentrations of IL-12p70, IL-23, IL-27, IFN- $\gamma$, IL-4 and IL-17A in the plasma were determined with specific FlowCytomix ${ }^{\mathrm{TM}}$ kits (eBioscience, San Diego, Calif., USA). The test samples were analyzed with a flow cytometer using a BD FACSAria instrument (Becton-Dickinson, San Jose, Calif., USA). The data for each cytokine were acquired using the FlowCytomix Pro 3.0 Software (eBioscience). For each analysis, up to 10,000 events were acquired. The minimum detectable concentrations of IL12p70, IL-23, IL-27, IFN- $\gamma$, IL-4 and IL-17A were $1.5,21.9,10,1.6$, 20.8 and $2.5 \mathrm{pg} / \mathrm{ml}$, respectively.

\section{Enzyme-Linked Immunosorbent Assay}

The plasma levels of IL-10 and IL-35 in cITP patients and healthy controls were also determined, using a commercial enzyme-linked immunosorbent assay kit (eBioscience) according to the manufacturer's instructions. The detection limit for this assay was 3.1 and $1.0 \mathrm{pg} / \mathrm{ml}$, respectively.

\section{Statistical Analysis}

Statistical analysis was performed with SPSS17.0 software (Chicago, Ill., USA). Data are presented as mean \pm standard deviation (SD) and median. Statistical significance of differences among the groups was determined with the nonparametric Mann-Whitney $\mathrm{U}$ test. A probability value of $\mathrm{p}<0.05$ was considered statistically significant. 
Table 1. Clinical characteristics and initial responses of cITP patients

\begin{tabular}{|c|c|c|c|c|c|}
\hline \multirow[t]{2}{*}{ Case No. } & \multirow[t]{2}{*}{ Sex/age, years } & \multirow{2}{*}{$\begin{array}{l}\text { Duration of ITP, } \\
\text { years }\end{array}$} & \multirow{2}{*}{$\begin{array}{l}\text { Bleeding } \\
\text { symptoms }\end{array}$} & \multicolumn{2}{|c|}{ Platelet count, $\times 10^{9} / 1$} \\
\hline & & & & $\begin{array}{l}\text { before HD-DXM } \\
\text { treatment }\end{array}$ & $\begin{array}{l}\text { after HD-DXM } \\
\text { treatment }\end{array}$ \\
\hline 1 & $\mathrm{M} / 58$ & 13 & PT, EC & 7 & 201 \\
\hline 2 & $\mathrm{~F} / 17$ & 1 & PT, EC & 11 & 15 \\
\hline 3 & $\mathrm{~F} / 36$ & 2 & $\mathrm{GH}$ & 5 & 142 \\
\hline 4 & $\mathrm{M} / 43$ & 2 & $\mathrm{PT}, \mathrm{EC}, \mathrm{GH}$ & 9 & 137 \\
\hline 5 & $\mathrm{~F} / 58$ & 5 & PT, EC & 18 & 79 \\
\hline 6 & $\mathrm{~F} / 29$ & 19 & $\mathrm{EC}$ & 13 & 145 \\
\hline 7 & $\mathrm{~F} / 23$ & 1 & $\mathrm{PT}, \mathrm{EC}$ & 28 & 117 \\
\hline 8 & $\mathrm{~F} / 54$ & 1 & PT, EC & 26 & 203 \\
\hline 9 & $\mathrm{~F} / 39$ & 6 & GUH & 5 & 57 \\
\hline 10 & $\mathrm{M} / 31$ & 18 & $\mathrm{GH}$ & 4 & 13 \\
\hline 11 & $\mathrm{~F} / 31$ & 10 & GUH & 9 & 22 \\
\hline 12 & $\mathrm{~F} / 18$ & 3 & $\mathrm{PT}, \mathrm{EC}$ & 20 & 53 \\
\hline 13 & $\mathrm{~F} / 62$ & 1 & PT, EC, GH & 4 & 133 \\
\hline 14 & $\mathrm{~F} / 71$ & 10 & $\mathrm{EC}$ & 6 & 158 \\
\hline 15 & $\mathrm{~F} / 43$ & 3 & EC & 12 & 66 \\
\hline 16 & $\mathrm{M} / 17$ & 2 & $\mathrm{EP}$ & 22 & 171 \\
\hline 17 & $\mathrm{M} / 54$ & 12 & PT, EC & 47 & 125 \\
\hline 18 & $\mathrm{~F} / 27$ & 6 & $\mathrm{EC}$ & 3 & 39 \\
\hline 19 & $\mathrm{M} / 37$ & 3 & $\mathrm{PT}$ & 27 & 163 \\
\hline 20 & $\mathrm{~F} / 72$ & 21 & $\mathrm{EC}, \mathrm{GH}$ & 14 & 19 \\
\hline 21 & $\mathrm{M} / 35$ & 1 & PT, EC & 8 & 125 \\
\hline 22 & $\mathrm{M} / 51$ & 21 & $\mathrm{PT}, \mathrm{EC}$ & 11 & 176 \\
\hline 23 & $\mathrm{~F} / 18$ & 2 & EC, GUH & 8 & 164 \\
\hline 24 & $\mathrm{~F} / 49$ & 7 & PT, EC, GH & 25 & 28 \\
\hline 25 & $\mathrm{~F} / 60$ & 15 & $\mathrm{PT}, \mathrm{EC}$ & 21 & 189 \\
\hline 26 & $\mathrm{~F} / 23$ & 3 & $\mathrm{PT}, \mathrm{EP}$ & 4 & 82 \\
\hline 27 & $\mathrm{~F} / 44$ & 9 & $\mathrm{PT}, \mathrm{EC}$ & 11 & 225 \\
\hline 28 & $\mathrm{M} / 35$ & 5 & PT, EC, EP & 12 & 19 \\
\hline 29 & $\mathrm{~F} / 21$ & 1 & GUH & 3 & 231 \\
\hline 30 & $\mathrm{M} / 39$ & 12 & $\mathrm{GH}$ & 34 & 155 \\
\hline 31 & $\mathrm{~F} / 47$ & 5 & PT, EC & 9 & 103 \\
\hline 32 & $\mathrm{~F} / 61$ & 6 & $\mathrm{EP}, \mathrm{GH}$ & 5 & 49 \\
\hline 33 & $\mathrm{~F} / 40$ & 4 & PT, GUH & 15 & 151 \\
\hline 34 & $\mathrm{M} / 41$ & 11 & $\mathrm{PT}, \mathrm{EP}$ & 11 & 15 \\
\hline 35 & $\mathrm{~F} / 27$ & 2 & GUH & 3 & 105 \\
\hline 36 & $\mathrm{M} / 36$ & 3 & $\mathrm{EC}$ & 2 & 76 \\
\hline 37 & $\mathrm{~F} / 23$ & 1 & PT, EC, GH & 21 & 208 \\
\hline 38 & $\mathrm{M} / 52$ & 7 & $\mathrm{EC}, \mathrm{GH}$ & 8 & 132 \\
\hline $\begin{array}{l}\text { Median } \\
(\min -\max )\end{array}$ & $39(17-72)$ & $5(1-21)$ & & $11(2-47)^{\mathrm{a}}$ & $125(13-231)^{\mathrm{b}}$ \\
\hline
\end{tabular}

$\mathrm{EC}=$ Ecchymoses; $\mathrm{EP}=$ epistaxis; $\mathrm{GH}$ = gingival hemorrhage; $\mathrm{GUH}$ = genitourinary hemorrhage; $\mathrm{PT}=$ petechiae. ${ }^{\mathrm{a}} \mathrm{p}<0.01$, cITP pretreatment vs. control. ${ }^{\mathrm{b}} \mathrm{p}<0.01$, cITP posttreatment vs. pretreatment.

\section{Results}

\section{Patient Characteristics}

The median age of the patients was 39 years and 32 years for controls. There were no significant differences in age and sex distribution between the cases and the controls ( $\mathrm{p}=0.198$ and 0.741 , respectively).

\section{Clinical Response to HD-DXM}

Of the 38 patients, a complete response was observed in $23(60.53 \%)$, a response in $8(20.05 \%)$ and no response in $7(18.42 \%)$. The median platelet count of the cITP patients before treatment was $11 \times 10^{9} / 1$ (range $2-47 \times$ $\left.10^{9} / 1\right)$. After HD-DXM treatment, this had increased significantly to a median of $125 \times 10^{9} / 1$ (range $13-231 \times$ 
Table 2. Plasma levels of cytokines in controls and in cITP patients before and after treatment, with a complete response, with a response and with no response

\begin{tabular}{|c|c|c|c|c|c|c|c|c|}
\hline & IL-12 & IL-23 & IL-27 & IL-35 & IFN $-\gamma$ & IL-4 & IL-17A & IL-10 \\
\hline Normal co & $14.73 \pm 5.95$ & $477.42 \pm 141.65$ & $32.91 \pm 20.37$ & $11.89 \pm 5.11$ & & & & \\
\hline Pretreatment $(n=38)$ & $27.38 \pm$ & $799.13 \pm 309.76^{\mathrm{a}}$ & $58.61 \pm 41.07^{\mathrm{a}}$ & $7.64 \pm 2.71^{\mathrm{a}}$ & $40.85 \pm 18.98^{a}$ & $57.36 \pm 16.35^{\mathrm{a}}$ & $28.77 \pm 25.78^{\mathrm{a}}$ & $148.59 \pm 54.26^{\mathrm{a}}$ \\
\hline Posttreatment $(\mathrm{n}=38)$ & $16.95 \pm 6.06^{\mathrm{b}}$ & $537.78 \pm 162.46^{\mathrm{b}}$ & $33.82 \pm 19.76^{\mathrm{b}}$ & $11.18 \pm 6.29^{\mathrm{b}}$ & $26.79 \pm 12.52^{b}$ & $76.64 \pm 20.76^{\mathrm{b}}$ & $12.42 \pm 8.39^{\mathrm{b}}$ & $221.32 \pm 71.42^{\mathrm{b}}$ \\
\hline A complete response $(n=23)$ & $13.73 \pm 4.35$ & $479.65 \pm 146.43$ & $30.58 \pm 13.37$ & $12.47 \pm 6.92$ & $23.28 \pm 11.73$ & $80.51 \pm 18.54$ & $8.91 \pm 4.20$ & $251.09 \pm 58.07$ \\
\hline A response $(\mathrm{n}=8)$ & $23.46 \pm 5.33^{c, d}$ & $561.93 \pm 124.08$ & $25.80 \pm 13.89$ & $10.24 \pm 6.12$ & $31.59 \pm 13.83^{\mathrm{d}}$ & $81.50 \pm 25.36$ & $20.05 \pm 8.60^{\mathrm{c}, \mathrm{d}}$ & $199.04 \pm 80.74$ \\
\hline No response $(n=7)$ & $20.07 \pm 4.07^{\mathrm{e}, \mathrm{f}}$ & $701.20 \pm 145.89^{\mathrm{e}, \mathrm{f}}$ & $53.60 \pm 30.75^{e, f}$ & $8.03 \pm 2.39^{\mathrm{e}, \mathrm{f}}$ & $32.81 \pm 10.85^{\mathrm{f}}$ & $58.37 \pm 12.96^{e, f}$ & $16.19 \pm 8.88^{e, f}$ & $148.99 \pm 36.31^{\mathrm{e}, \mathrm{f}}$ \\
\hline
\end{tabular}

Values are given as $\mathrm{pg} / \mathrm{ml} .{ }^{a} \mathrm{p}<0.01$, cITP pretreatment vs. normal controls. ${ }^{\mathrm{b}} \mathrm{p}<0.01$, cITP posttreatment vs. pretreatment. ${ }^{\mathrm{c}} \mathrm{p}<0.05$, a response vs. a complete response. ${ }^{\mathrm{d}} \mathrm{p}<0.05$, a response vs. normal controls. ${ }^{\mathrm{e}} \mathrm{p}<0.05$, no response vs. a complete response. ${ }^{\mathrm{f}} \mathrm{p}<0.05$, no response vs. normal controls.

$\left.10^{9} / 1\right)$. The difference was statistically significant $(\mathrm{p}<$ 0.01 ; table 1 ). No bleeding or other obvious complications were observed during treatment.

\section{Cytokine Changes in cITP Patients}

The cytokine levels in the cITP patients and controls are presented in table 2 . The plasma levels of IL-12p70, IL-23, IL-27, IFN- $\gamma$ and IL-17A were significantly higher in the cITP patients than in the controls before HD-DXM treatment ( $p<0.01$; fig. 1). Conversely, the levels of IL-4, IL-10 and IL-35 were considerably lower in the patients than in the controls $(\mathrm{p}<0.01$; fig. 1$)$. The plasma levels of IL-12p70 ( $\mathrm{r}=-0.586, \mathrm{p}<0.001)$, IL-23 ( $\mathrm{r}=-0.498, \mathrm{p}=$ $0.001)$ and IFN $-\gamma(\mathrm{r}=-0.384, \mathrm{p}=0.017)$ were negatively correlated with the platelet counts in the cITP patients before HD-DXM treatment.

\section{Cytokine Profile Correction after Treatment}

The plasma levels of IL-12p70, IL-23, IL-27, IFN- $\gamma$ and IL-17A in patients had decreased significantly after treatment ( $\mathrm{p}<0.01$; fig. 1 ) and normalized after HD-DXM treatment, while the plasma levels of IL-4, IL-10 and IL35 had increased significantly ( $p<0.01$; fig. 1 ) after the treatment. There were no significant differences in the levels of the above cytokines between the posttreatment patients and the normal controls $(\mathrm{p}>0.05)$. The plasma levels of IL-12p70 ( $r=-0.535, \mathrm{p}<0.001)$, IL-23 ( $\mathrm{r}=$ $-0.567, \mathrm{p}<0.001)$ and IL-17 $(\mathrm{r}=-0.481, \mathrm{p}=0.002)$ were negatively correlated with the platelet counts in the cITP patients after treatment. Conversely, levels of IL-4 ( $\mathrm{r}=$ $0.395, \mathrm{p}=0.014)$ and IL-10 $(\mathrm{r}=0.412, \mathrm{p}=0.010)$ were positively correlated with platelet counts.

There were no significant differences in the plasma levels of cytokines between the patients with a complete response and the normal controls $(\mathrm{p}>0.05)$. However, the levels of IL-12p70, IL-23, IL-27, IFN- $\gamma$ and IL-17A were still significantly higher in patients with no response than in normal controls, and IL-4, IL-10 and IL-35 levels were significantly lower in the patients with no response ( $\mathrm{p}<0.05$; fig. 1).

\section{Discussion}

The pathophysiology of ITP is heterogeneous and complex [22]. Although a few of the cytokines associated with ITP have been studied previously, the profile of cytokines in ITP is still controversial [23]. In this particular report, the plasma levels of IL-12p70, IL-23, IL-27, IFN- $\gamma$ and IL-17A were significantly higher in cITP patients than those in the controls before HD-DXM treatment. Conversely, the levels of IL-4, IL-10 and IL-35 patients were considerably lower than in the controls. IL-12 and IL-23 can induce or promote the generation of proinflammatory Th1 and Th17 cells involved in the progress of cITP $[2,4]$. Our study showed that the levels of IL-12 and IL-23 were upregulated in the pretreatment cITP patients compared to in the healthy controls. After HDDXM treatment, however, the levels of IL-12 and IL-23 had decreased; this confirms previous reports [24]. Hence, both of these cytokines could also be pathogenic factors of cITP. Moreover, we found that the plasma levels of IL12 and IL-23 were negatively correlated with the platelet counts of cITP patients before and after treatment, suggesting that the expression of IL-12 and IL-23 was correlated with the disease activity. IL-27 is an immunoregulatory cytokine, but it may have some prostimulatory functions [10-12]. We recently identified that IL-27 was increased in patients with active CITP, and that it can cause Th1 differentiation [25]. In this study, we observed a dramatically decreased plasma IL-27 level in patients with cITP after HD-DXM treatment. A decrease in Treg 

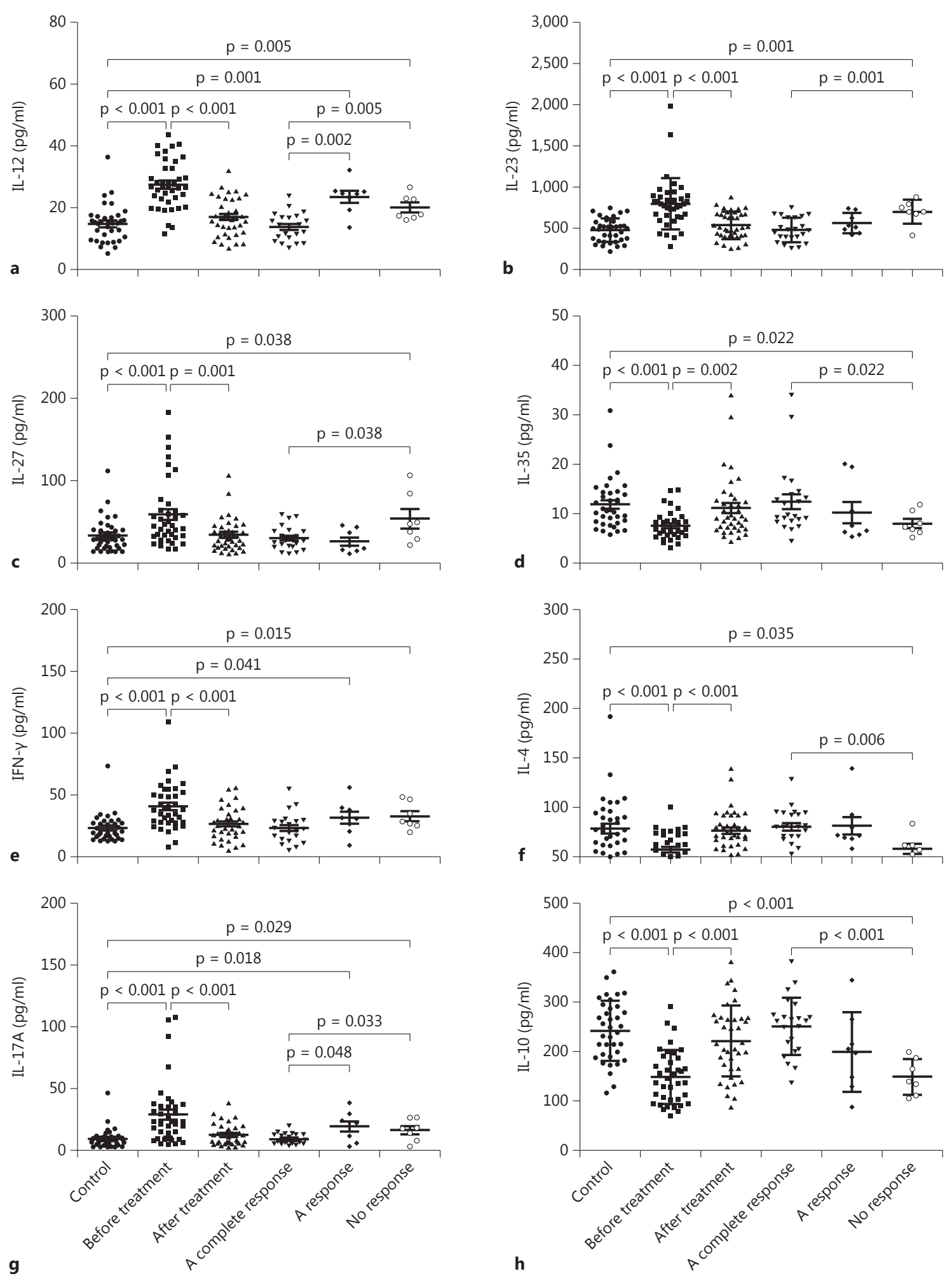

Fig. 1. Comparison of IL-12 (a), IL-23 (b), IL-27 (c), IL-35 (d), IFN- $\gamma$ (e), IL-4 (f), IL-17A (g) and IL-10 (h) plasma levels in healthy controls and in cITP patients before treatment, after HD-DXM treatment, a complete response, a response and no response. 
cell numbers and functioning was displayed in the cITP patients [3]. As IL-35 is considered to be the characteristic factor of Treg cells, it could elicit the development of iTr35 cells, a population of induced Treg cells that suppress via IL-35 but do not express Foxp3, IL-10 or TGF- $\beta$. The deficiency of IL-35 might lead to the dysfunction of self-tolerance that contributes to the platelet destruction.

Subsets of immune cells and the cytokines they produce play a key role in ITP [26]. It has been reported that an increased Th1/Th2 ratio in the peripheral blood appears to be a key pathogenic mechanism of cITP [2]. In addition, recent studies have indicated that upregulation of Th17 cells may be an important determinant in the evolution of cITP [4]. In agreement with previous reports $[2,4]$, the plasma levels of IFN- $\gamma$, IL- 4 and IL-17A in our patients also implicate a Th1 and Th17-dominated cytokine profile in cITP.

In our study, the $80.58 \%$ of the responses of patients to 4 days of HD-DXM treatment confirmed the results of previous reports [17, 18, 21]. Borst et al. [18] reported that HD-DXM led to an acute response (a rise in platelet count to $>50 \times 10^{9} / 1$ ) in $83 \%$ and also side effects that were frequent but rarely dangerous. Cheng et al. [21] reported that a good initial response to HD-DXM occurred in $106 / 125$ patients (85\%). HD-DXM might be a promising alternative to prednisone as a first- or second-line treatment for ITP patients $[18,21]$. However, the specific mechanism of DXM regulation of cytokine levels has not been completely clarified. It has been reported that GCs inhibit the transcription factors of the NF- $\kappa \mathrm{B}$ and AP-1 families [27], and thus suppress the production of multiple cytokines including IL-2, IL-6, IFN- $\gamma$, IL-23 and IL-8 [28]. Moreover, the interaction of GCs with the Jak-STAT signaling pathway may have either a positive or a negative impact on cytokine signaling. One example of a positive interaction is the synergistic activation of $\beta$-casein gene expression in the mammary gland, mediated by a direct physical interaction between prolactin-activated STAT5 and the GC receptor, which is activated by GCs [29]. In T lymphocytes, DXM reduces cellular responsiveness to IL12 and inhibits IL-12-induced STAT4 activation without altering the phosphorylation of Jaks [30]. Furthermore, we found that the IL-12 family cytokines and their relevant cytokine levels in patients who attained a complete response returned to the levels of the normal controls, but that these levels were not corrected in the patients with no response. These data indicated that the patients with cITP who had not responded were rooted in a cytokine imbalance, and may have needed additional treatment. However, further study is necessary to determine the precise mechanisms of HD-DXM regulation of the cytokine secretion profile in patients with cITP.

\section{Conclusion}

We demonstrated that abnormal expression of the IL12 family cytokines and their relevant cytokine levels in patients with cITP was corrected with HD-DXM treatment. Significant variations in cytokine levels according to the clinical response could help to improve the understanding of the etiology, evolution and therapeutics of the disease. Future studies are necessary to research the signaling pathways associated with cytokines, in order to shed light on the molecular mechanisms of cITP.

\section{Acknowledgments}

This work was supported by research grants from the Higher Education Department of Anhui Province (KJ2011A167, KJ2011Z165) and the Anhui Technological Development Project (1208085MH154).

\section{Disclosure Statement}

The authors have no conflicts of interest to disclose.

\section{References}

1 McMillan R: Chronic idiopathic thrombocytopenic purpura. N Engl J Med 1981;304: $1135-1147$.

2 Ogawara H, Handa H, Morita K, et al: High Th1/Th2 ratio in patients with chronic idiopathic thrombocytopenic purpura. Eur J Haematol 2003;71:283-288.

IL-12 Family Cytokines in cITP Patients during HD-DXM Therapy
Yu J, Heck S, Patel V, et al: Defective circulating CD25 regulatory $\mathrm{T}$ cells in patients with chronic immune thrombocytopenic purpura. Blood 2008;112:1325-1328.

4 Rocha AM, Souza C, Rocha GA, et al: The levels of IL-17A and of the cytokines involved in Th17 cell commitment are increased in patients with chronic immune thrombocytopenia. Haematologica 2011;96:1560-1564.

\footnotetext{
5 Olsson B, Andersson PO, Jernås M, et al: Tcell-mediated cytotoxicity toward platelets in chronic idiopathic thrombocytopenic purpura. Nat Med 2003;9:1123-1124.

6 Vignali DA, Kuchroo VK: IL-12 family cytokines: immunological playmakers. Nat Immunol 2012;13:722-728.
} 
7 Trinchieri G: Interleukin-12 and the regulation of innate resistance and adaptive immunity. Nat Rev Immunol 2003;3:133-146.

8 Morrison PJ, Ballantyne SJ, Kullberg MC: Interleukin-23 and T helper 17-type responses in intestinal inflammation: from cytokines to T-cell plasticity. Immunology 2011;133:397408.

9 Pflanz S, Timans JC, Cheung J, et al: IL-27, a heterodimeric cytokine composed of EBI3 and $\mathrm{p} 28$ protein, induces proliferation of naive CD4+ T cells. Immunity 2002;16:779-790.

10 Awasthi A, Carrier Y, Peron JP, et al: A dominant function for interleukin 27 in generating interleukin 10-producing anti-inflammatory T cells. Nat Immunol 2007;8:1380-1389.

11 Yoshimoto T, Yoshimoto T, Yasuda K, et al: IL-27 suppresses Th2 cell development and Th2 cytokine production form polarized Th2 cells: a novel therapeutic way for Th2-mediated allergic inflammation. J Immunol 2007; 179:4415-4423.

12 Diveu C, McGeachy MJ, Boniface K, et al: IL27 blocks RORc expression to inhibit lineage commitment of Th17 cells. J Immunol 2009; 182:5748-5756.

13 Collison LW, Workman CJ, Kuo TT, et al: The inhibitory cytokine IL-35 contributes to regulatory T-cell function. Nature 2007;450: 566-569.

14 Whitehead GS, Wilson RH, Nakano K, et al: IL-35 production by ICOS+ regulatory T cells reverses established IL-17-dependent allergic airways disease. J Allergy Clin Immunol 2012 129:207-215
15 Chaturvedi V, Collison LW, Guy CS, et al: Cutting edge: human regulatory $\mathrm{T}$ cells require IL-35 to mediate suppression and infectious tolerance. J Immunol 2011;186:66616666.

16 Bettini M, Castellaw AH, Lennon GP, et al: Prevention of autoimmune diabetes by ectopic pancreatic $\beta$-cell expression of interleukin-35. Diabetes 2012;61:1519-1526.

17 Mazzucconi MG, Fazi P, Bernasconi S, et al: Therapy with high-dose dexamethasone (HD-DXM) in previously untreated patients affected by idiopathic thrombocytopenic purpura: a GIMEMA experience. Blood 2007; 109:1401-1407.

18 Borst F, Keuning JJ, van Hulsteijn $\mathrm{H}$, et al: High-dose dexamethasone as a first- and second-line treatment of idiopathic thrombocytopenic purpura in adults. Annu Hematol 2004;83:764-768

19 Guo C, Chu X, Shi Y, et al: Correction of Th1dominant cytokine profiles by high-dose dexamethasone in patients with chronic idiopathic thrombocytopenic purpura. J Clin Immunol 2007;27:557-562.

20 Rodeghiero F, Stasi R, Gernsheimer T, et al: Standardization of terminology, definitions and outcome criteria in immune thrombocytopenic purpura of adults and children: report from an international working group. Blood 2009;113:2386-2393.

21 Cheng Y, Wong RS, Soo YO, et al: Initial treatment of immune thrombocytopenic purpura with high-dose dexamethasone. N Engl J Med 2003;349:831-836.

22 Stasi R, Evangelista ML, Stipa E, et al: Idiopathic thrombocytopenic purpura. Current concepts in pathophysiology and management. Thromb Haemost 2008;99:4-13.
23 Zhan Y, Zou S, Hua F, et al: High-dose dexamethasone modulates serum cytokine profile in patients with primary immune thrombocytopenia. Immunol Lett 2014;160:33-38.

24 Liu H, Ouyang X, Li Y, et al: Involvement of levels of Toll like receptor-4 in monocytes, CD4+ T-lymphocyte subsets, and cytokines in patients with immune thrombocytopenic purpura. Thromb Res 2013;132:196-201.

25 Li Q, Zhang L, Yang M, et al: Increased interleukin-27 promotes Th1 differentiation in patients with chronic immune thrombocytopenia. Scand J Immunol 2014:80:276-282.

26 Talaat RM, Elmaghraby AM, Barakat SS, et al: Alteration in immune cell subsets and their cytokine secretion profile in childhood idiopathic thrombocytopenic purpura (ITP). Clin Exp Immunol 2014;176:291-300.

27 Karin M: New twists in gene regulation by glucocorticoid receptor: is DNA binding dispensable? Cell 1998;93:487-490.

28 Wilckens T, De Rijk R: Glucocorticoids and immune function: unknown dimensions and new frontiers. Immunol Today 1997; 18:418424

29 Stöcklin E, Wissler M, Gouilleux F, et al: Functional interactions between Stat 5 and the glucocorticoid receptor. Nature 1996;383: 726-728.

30 Wu CY, Wang K, McDyer JF, et al: Prostaglandin E2 and dexamethasone inhibit IL-12 receptor expression and IL-12 responsiveness. J Immunol 1998;161:2723-2730. 